\title{
Query optimization using rewrite rules
}

Sieger van Denneheuvel (UvA)

Karen Kwast (UvA)

Gerard R. Renardel de Lavalette (RUU)

Edith Spaan (UvA)

Logic Group

Preprint Series

No. 58

October 1990
Department of Philosophy

University of Utrecht

Heidelberglaan 2

3584 CS Utrecht

The Netherlands 


\title{
Query optimization using rewrite rules
}

\author{
Sieger van Denneheuvel (UVA) \\ Karen Kwast (UVA) \\ Gerard R. Renardel de Lavalette (RUU) \\ Edith Spaan (UVA) \\ Department of Mathematics and Computer Science \\ University of Amsterdam (UVA) \& \\ Applied Logic Group, Department of Philosophy \\ University of Utrecht (RUU)
}

\begin{abstract}
In this paper we present a normal form for a relational algebra, consisting of Projection, Selection and Join, extended with Calculation and Union and define a corresponding language UPCSJL. The construction of this normal form, using unconditional rewrite rules, already provides some optimization. Further optimization can be achieved efficiently by applying conditional rewrite rules that directly operate on the normal form. This approach is compared to more traditional query optimization techniques that do not apply normalization.
\end{abstract}

\section{Introduction}

PSJ expressions are relational algebra expressions containing only project, select and join operators. This restricted class of expressions, called PSJL in the sequel, is commonly used in relational databases. PSJL expressions are studied in [YAN87] and [LAR85], where it is mentioned without proof (which is not very difficult) that they can be reduced into a normal form where first the join operators are applied, then selection and finally projection. Such normalization procedures play an important role in query optimization: see [ULL89] and [YAN87]. Standard optimization techniques can be used to further optimize PSJL normal form expressions: e.g. in special circumstances the 'selection before join' heuristic can be applied to push selection down to the relational database tables ([ULL89]).

There are several reasons to apply normalization before optimization. Firstly, normalization reduces the number of relational operators in a relational expression. As a consequence, optimization after normalization can be more efficient since the number of reducible subexpressions (redexes) on which optimization rules are applied is also reduced. Moreover the optimization rules can benefit from the fixed structure of a normal form.

Secondly there is a functional difference between rules used for normalization and rules used for optimization: the former are unconditional whereas the latter are conditional. A normalization rule should always be applicable on a subexpression of the proper syntactical format or else a normal form could not be obtained. On the other hand optimization rules only rewrite if in addition to a proper format also a condition involving the subexpression is satisfied. Therefore optimization is in general more expensive than normalization, since applications of optimization rules may fail.

In this paper we add the relational operators calculate and union to the above mentioned relational operators, thus obtaining the language of UPCSJL expressions. The question arises whether UPCSJL expressions also can be reduced into a normal form. We prove the existence of a normal form, where the joins are followed by selection, calculation, projection and finally union; moreover, our proof yields a direct construction. This UPCSJL normal form already performs some optimization, but the normal form procedure can serve as the starting point for further optimization (just as for PSJL normal forms). 
Our interest for UPCSJL expressions lies in its role in the integration of relational databases and constraint solving. This integration is one of the aims of the declarative Rule Language RL. The potential for such an integration has been investigated in the context of the Rules Technology project led by Peter Lucas at the IBM San Jose Research Center: see e.g. [HANS89], [HANS88]. RL was defined by Peter van Emde Boas in [VEMD86a], where a relational semantic model is given to interpret RL (see also [VEMD86b], [VEMD86c]). A considerable part of this language has been implemented: see [DEN90a] and [DEN90b].

RL can be considered as an extension of SQL with existential quantification over variables occurring in constraints but not necessarily in relations (as is required in SQL, where all variables in the WHERE clause have to be present in the FROM clause; see [DATE89]). As a consequence, not all expressions in RL can be evaluated: imagine what happens when the existential quantifier ranges over an infinite domain. To be able to deal with these problems, the above-mentioned implementation of RL is equipped with a constraint solver, which transforms evaluable RL expressions into expressions of UPCSJL.

Acknowledgements. The authors thank Peter van Emde Boas (University of Amsterdam) for useful criticism and remarks.

\section{The data language $\mathrm{DL}$}

We begin with the definition of the data language DL: it will act as a parameter for the language UPCSJL. DL is a many-sorted language containing variables (denoted by the metavariables $x, y, \ldots$, also called attributes), constants $(c, d, \ldots$, also called values), functions $(f, g, \ldots),=$ (the equality predicate), predicates, propositional connectives $(\neg, \wedge, \vee, \rightarrow)$ and the propositional constant true. Terms $(s, t, \ldots)$ and assertions $(A, B, \ldots$, also called constraints or conditions $)$ are defined as usual.

If $E$ is any of the items defined above (or a collection of these), then $\operatorname{var}(E)$ is the set of variables occurring in $E$. Furthermore, we assume some evaluation mechanism eval(-) for DL to be given, which evaluates closed terms (terms without variables) to constants and closed assertions to truth values.

We give an example language for $\mathbf{D L}$, defined by the following sorts, constants, functions and predicates:

sorts: NUM (natural numbers), STR (strings of characters)

constants: $0,1,2, \ldots$ in NUM, all finite strings in STR

functions:

$*,+: \mathbf{N U M} \times \mathbf{N U M} \rightarrow \mathbf{N U M}$

cat: $\mathbf{S T R} \times \mathbf{S T R} \rightarrow \mathbf{S T R}$ (concatenation)

length: STR $\rightarrow$ NUM (length of a string)

digits: NUM $\rightarrow$ STR (converts a number to its string representation)

predicates: $<,>, \leq, \geq, \neq$ (binary predicates, both on NUM and on STR)

\section{Functions of the language UPCSJL}

Before we define the sorts of the language UPCSJL, we introduce the following.

Definition $1 A$ solution is an expression of the form $x=t$ with $x \notin \operatorname{var}(t)$.

Definition $2 A$ solution set is a finite set $\left\{x_{1}=t_{1}, \ldots, x_{n}=t_{n}\right\}$, satisfying:

1. $\left\|\left\{x_{1}, \ldots, x_{n}\right\}\right\|=n$, (the variables are distinct)

2. $\left\{x_{1}, \ldots, x_{n}\right\} \cap \operatorname{var}\left(\left\{t_{1}, \ldots, t_{n}\right\}\right)=\emptyset$

A tuple (denoted by $\phi, \psi, \ldots$ ) is a solution set of the form $\left\{x_{1}=c_{1}, \ldots, x_{n}=c_{n}\right\}$. For tuples $\phi$, we often write $\operatorname{attr}(\phi)$ instead of $\operatorname{var}(\phi)$. Tuples are called similar if they have the same attributes. A relation $\mathrm{R}$ is a pair $\left\langle X, R^{\prime}>\right.$ of a finite collection of attributes $X$ and a finite collection of similar tuples, satisfying:

$$
\forall \phi \in R^{\prime} \operatorname{attr}(\phi)=X
$$


If $R^{\prime}$ is non-empty then $X$ can be obtained from $R^{\prime}$; since most relations are nonempty, we shall allow ourselves to be a bit sloppy and identify $R$ and $R^{\prime}$; i.e. consider a base relation to be a collection of similar tuples.

Example 1 (solution sets and tuples)

$\{$ name $=$ 'bob', age $=55$, dep $=$ 'toy $\}$ (a tuple)

$\{x=1, y=2, z=3\}$ (a tuple)

$\{x=u+2, y=v+2\}$ (a solution set)

Assume that an instance of $\mathbf{D L}$ is given, i.e. some language with sorts, variables, constants, etc. We now present the functions used in the definition of the language UPCSJL. UPCSJL is a four-sorted language with expressions (thus named to distinguish them from DL-terms) and equations. The sorts are:

- $\mathcal{V}$ (finite sets of DL-variables)

- $\mathcal{C}$ (constraints, i.e. DL-assertions)

- $\mathcal{S}$ (solutions sets)

- $\mathcal{R}$ (relations)

We let $X, Y, Z$ range over $\mathcal{V} ; A, B, C$ over $\mathcal{C} ; \Phi, \Psi$ over $\mathcal{S} ; R, S$ over $\mathcal{R}$. An expression of sort $\mathcal{R}$ is also called a relational expression. A base relation is sometimes followed by a bracketed list denoting all its attributes. In the sequel we use the notation $E^{X}$ for renaming an expression $E$ with respect to a variable set $X$ :

\section{Definition 3}

$E^{X}=E^{\prime}$ where $E^{\prime}$ is obtained by renaming all variables $\operatorname{var}(E)-X$ uniquely.

To allow a brief notation, a renaming $(-)^{X}$ can be applied at several places in an expression. In that case all occurrences of $(-)^{X}$ denote the same renaming:

Example 2 (renaming variables in expressions with $X=\{x\}, Y=\{y\}$ )

$\sigma\left((r(x, y))^{X} \bowtie s(y, z)^{Y},(x>y)^{X} \wedge(y>z)^{Y}\right) \rightarrow \sigma\left(r\left(x, u_{1}\right) \bowtie s\left(y, u_{2}\right), x>u_{1} \wedge y>u_{2}\right)$

Next we present the functions of UPCSJL. They are grouped according to their range.

\subsection{Functions with range $\mathcal{V}$}

Besides the usual set operations $\cup, \cap$ and -, we have:

Definition 4 (attributes of a relational expression)

$\operatorname{attr}(-): \mathcal{R} \rightarrow \mathcal{V}$

Definition 5 (head and tail variables of a solution set)

$h \operatorname{var}(-): \mathcal{S} \rightarrow \mathcal{V}$

$\operatorname{tvar}(-): \mathcal{S} \rightarrow \mathcal{V}$

$h \operatorname{var}\left(\left\{x_{1}=t_{1}, \ldots, x_{n}=t_{n}\right\}\right)=\left\{x_{1}, \ldots, x_{n}\right\}$

$\operatorname{tvar}\left(\left\{x_{1}=t_{1}, \ldots, x_{n}=t_{n}\right\}\right)=\operatorname{var}\left(\left\{t_{1}, \ldots, t_{n}\right\}\right)$

\subsection{Functions with range $\mathcal{C}$}

Besides $\wedge$ (conjunction), we have the merge of two solution sets, yielding a constraint. The merge function is defined as follows:

Definition $6 \oplus: \mathcal{S} \times \mathcal{S} \rightarrow \mathcal{C}$

$\Phi \oplus \Psi=\{s=t \mid x=s \in \Phi, x=t \in \Psi\}$

Example 3 (merging solution sets)

$\{x=' b o b '\} \oplus\{x=y$ cat $z\}=\left\{{ }^{\prime} b o b '=y\right.$ cat $\left.z\right\}$

$\{x=u+2, y=3\} \oplus\{x=v+2\}=\{u+2=v+2\}$

$\{x=y+2\} \oplus\{x=z, y=2 * z\}=\{y+2=z\}$

$\{x=y+2\} \oplus\{z=x, y=2 * x\}=\emptyset$ 
Solution sets $\Phi=\left\{x_{1}=t_{1}, \ldots, x_{n}=t_{n}\right\}$ can be interpreted as substitutions $\left[x_{1}:=t_{1}, \ldots, x_{n}:=t_{n}\right]$ which can be applied to (collections of) items. So we have an operation apply:

Definition 7 -(-) $: \mathcal{S} \times \mathcal{C} \rightarrow \mathcal{C}$

$\Phi(A)=A\left[x_{1}:=t_{1}, \ldots, x_{n}:=t_{n}\right]$ with $\Phi=\left\{x_{1}=t_{1}, \ldots, x_{n}=t_{n}\right\}$

Example 4 (substitution on constraints)

$\{x=u+2\}(\{x=u+1\})=\{u+2=u+1\}$

$\{x=u+2, y=v+2\}(\{x>y\})=\{u+2>v+2\}$

\subsection{Functions with range $\mathcal{S}$}

Here, too, we have the usual set operations $\cup, \cap$ and - ; besides, we introduce the restrict and delete functions:

Definition 8 (restricting and deleting solutions)

1. [-] $: \mathcal{S} \times \mathcal{V} \rightarrow \mathcal{S}$

$\Phi[X]=\{x=t \in \Phi \mid x \in X\}$

2. $-\langle-\rangle: \mathcal{S} \times \mathcal{V} \rightarrow \mathcal{S}$

$\Phi\langle X\rangle=\{x=t \in \Phi \mid x \notin X\}$

Example 5 (restriction and deletion)

$\{x=z+1, y=z+2\}[\{x\}]=\{x=z+1\}$

$\{x=z+1, y=z+2\}\langle\{x\}\rangle=\{y=z+2\}$

$\{x=1, y=2, z=3\}\langle\{x\}\rangle[\{y\}]=\{y=2\}$

Further we also have substitution on solutions:

Definition $9-((-)): \mathcal{S} \times \mathcal{S} \rightarrow \mathcal{S}$

$\Phi((\Psi))=\{x=\operatorname{eval}(\Phi(t)) \mid x=t \in \Psi\}$

Example 6 (substitution on solution sets)

$\{x=u+2\}((\{x=u+1\}))=\{x=u+1\}$

$\{u=2\}((\{x=u+1\}))=\{x=3\}$

$\{x=u+2, y=v+2\}((\{x=u+1,3=y\}))=\{x=u+1,3=v+2\}$

\subsection{Functions with range $\mathcal{R}$}

Here we find the usual operators on relations, together with the calculate operator. The definitions are:

Definition 10 (primitive relational operators)

1. $\pi: \mathcal{R} \times \mathcal{V} \rightarrow \mathcal{R}$

$\pi(R, X)=\{\phi[X] \mid \phi \in R\} \quad$ if $X \subset \operatorname{attr}(R)$

2. $\sigma: \mathcal{R} \times \mathcal{C} \rightarrow \mathcal{R}$

$\sigma(R, A)=\{\phi \in R \mid \operatorname{eval}(\phi(A))=$ true $\} \quad$ if $\quad \operatorname{var}(A) \subset \operatorname{attr}(R)$

3. $\kappa: \mathcal{R} \times \mathcal{S} \rightarrow \mathcal{R}$

$\kappa(R, \Phi)=\{\psi \cup \psi((\Phi)) \mid \psi \in R\} \quad$ if $\quad \operatorname{tvar}(\Phi) \subset \operatorname{attr}(R)$ and $h \operatorname{var}(\Phi) \cap \operatorname{attr}(R)=\emptyset$

4. $-\bowtie_{-}: \mathcal{R} \times \mathcal{R} \rightarrow \mathcal{R}$

$R \bowtie S=\{\phi \cup \psi \mid \phi \in R, \psi \in S, \forall x \in \operatorname{attr}(\phi) \cap \operatorname{attr}(\psi)(\phi[x]=\psi[x])\}$

5. $-\cup_{-}: \mathcal{R} \times \mathcal{R} \rightarrow \mathcal{R}$

$R \cup S=\{\phi \mid \phi \in R \vee \phi \in S\} \quad$ if $\operatorname{attr}(R)=\operatorname{attr}(S)$

One readily observes that the project, select, calculate and union operators are partial since they are only defined when certain conditions on the arguments are met. These conditions are referred to as wellformedness conditions. They are quite reasonable: the wellformedness condition for projection ensures that a relation is not projected on attributes that are not part of the relation; the wellformedness condition for selection takes care that the constraint $A$ can indeed be evaluated to true or false; the first part of the wellformedness condition for the calculate operator ensures that the tails of solutions in $\Phi$ can be evaluated, the second part rules out the possibility that the head of a solution is also determined directly by an attribute of the relation $R$. The constraint set $A$ in $\sigma(R, A)$ is the condition of the select operator and the solution set $\Phi$ in $\kappa(R, \Phi)$ is the computation of the calculate operator. 
Example 7 (extending tuples with the calculate operator)

$r(x, y):=\{\{x=1, y=2\}\}$

$\kappa(r(x, y),\{u=x+y, v=' b o b '\})=\{\{x=1, y=2, u=3, v=' b o b '\}\}$

\section{Relational rewrite rules}

In this section we describe a large number of rules handling the properties of the primitive relational operators. Since these operators involve wellformedness conditions we introduce a special notation to emphasize in what direction a rule can be applied:

Definition 11 (notation for rewriting)

1. $R \rightarrow S$ iff $R$ is wellformed $\Rightarrow S$ is wellformed \& $R=S$

2. $R \leftrightarrow S \quad$ iff $R \rightarrow S \& S \rightarrow R$

For some rewrite rules $R \rightarrow S$, wellformedness of $R$ does not imply wellformedness of all subexpressions of $S$ and in this case the wellformedness conditions on the violating subexpressions are represented in the rewrite rule as a condition.

\subsection{Projection, selection and calculation}

The projection, selection and calculate operators have in common that they are applied on a single relational argument and therefore we discuss them together in this section. In the sequel we cluster rules that are similar in one proposition if possible.

Proposition 12 (cascade rules)

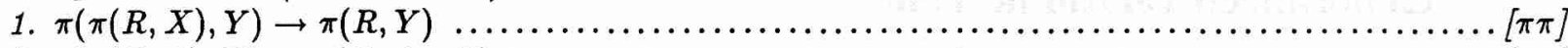

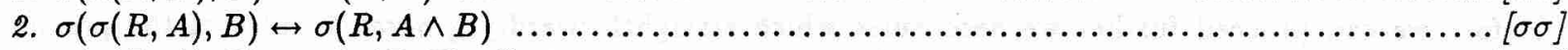

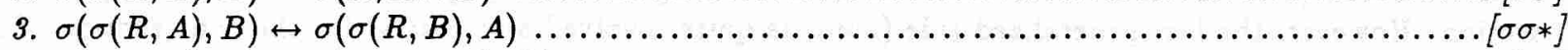

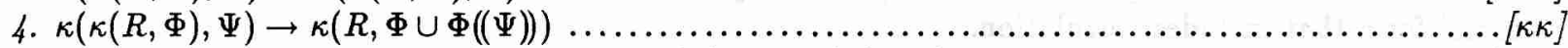

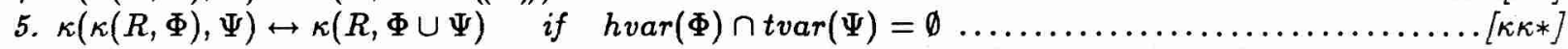

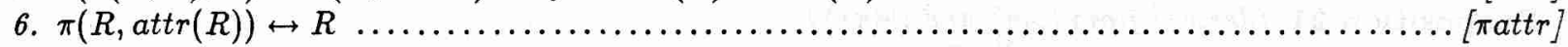

Proposition 13 (selection and projection)

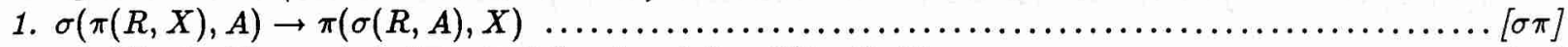

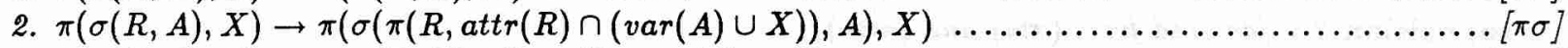

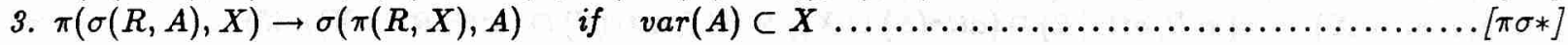

Proposition 14 (selection and calculation)

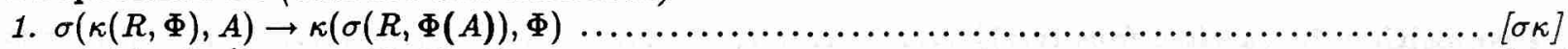

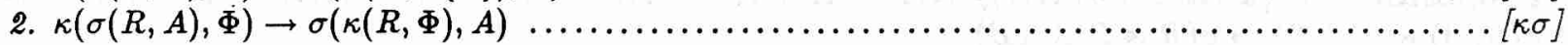

Proposition 15 (calculation and projection)

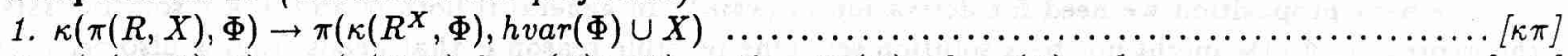

2. $\kappa(\pi(R, X), \Phi) \rightarrow \pi(\kappa(R, \Phi), h \operatorname{var}(\Phi) \cup X)$ if $\operatorname{attr}(R) \cap h \operatorname{var}(\Phi)=\emptyset \ldots \ldots \ldots \ldots \ldots \ldots[\kappa \pi]$

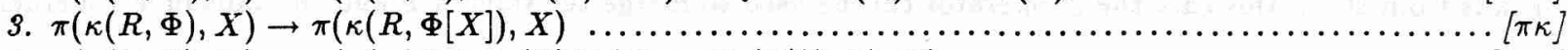

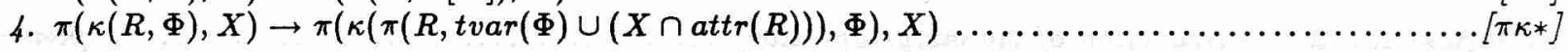

Note that in the rule $(\kappa \pi)$ by the renaming in $R^{X}$ and the wellformedness of $\kappa(\pi(R, X), \Phi)$ all variables $h \operatorname{var}(\Phi)$ are different from the variables $\operatorname{attr}\left(R^{X}\right)$, as required.

\subsection{Join and union}

In this section we add the join and union operators in our list of rules. As before some rules have both conditional and unconditional versions.

Proposition 16 (symmetry and associativity of $\bowtie$ and $U$ )

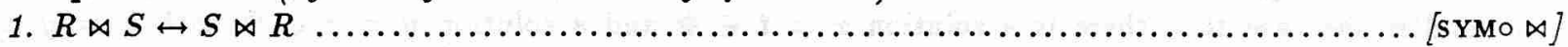

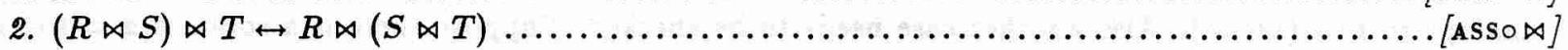

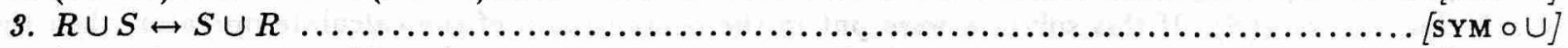

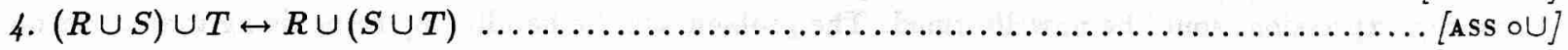


Proposition 17 (join and calculation)

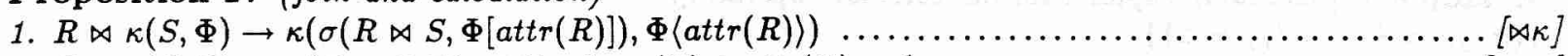

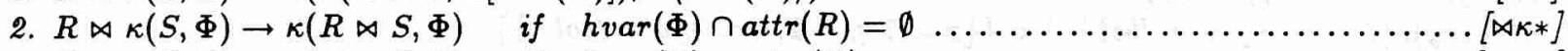

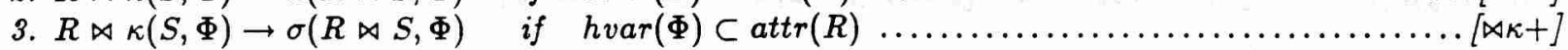

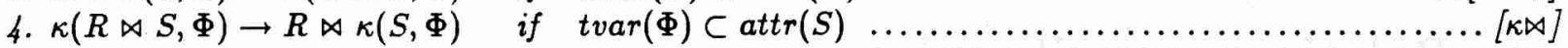

Proposition 18 (join and projection)

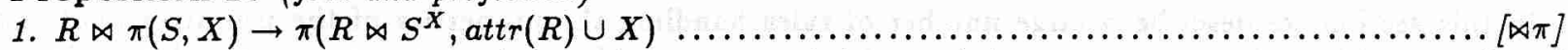

2. $R \bowtie \pi(S, X) \rightarrow \pi(R \bowtie S, \operatorname{attr}(R) \cup X)$ if $\operatorname{attr}(R) \cap \operatorname{attr}(S) \subset X \ldots \ldots \ldots \ldots \ldots \ldots \ldots \ldots \ldots \pi]$

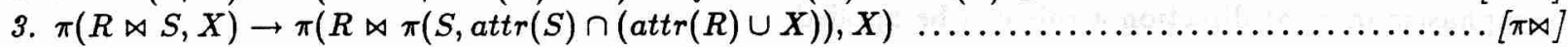

4. $\pi(R \bowtie S, X) \rightarrow \pi(R \bowtie \pi(S, \operatorname{attr}(S) \cap X), X) \quad$ if $\quad \operatorname{attr}(R) \cap \operatorname{attr}(S) \subset X \ldots \ldots \ldots \ldots \ldots \ldots[\pi \bowtie *]$

Proposition 19 (join and selection)

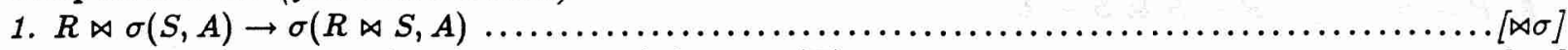

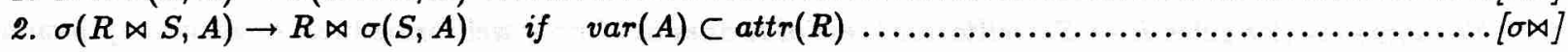

Proposition 20 (distribution rules for union)

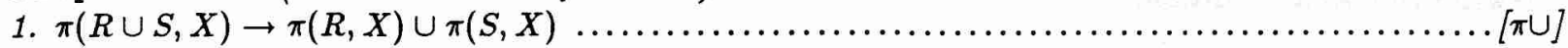

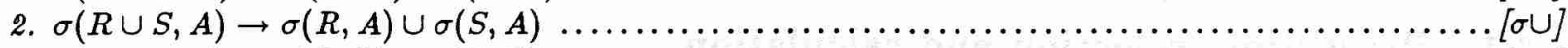

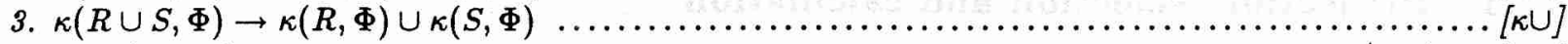

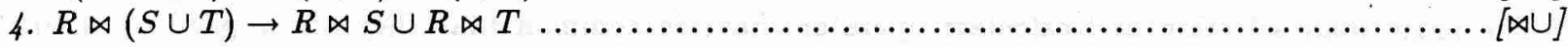

Distribution rules for the join operator are described in the next section.

\subsection{Generalized relational rules}

Before we can proceed further we need rules which straightforwardly generalize rules of the previous section. However, the last generalized rule $(\kappa \bowtie \kappa)$ is quite involved and crucial for the construction of a normal form that includes calculation.

Proposition 21 (derived from $(\bowtie \pi)$ and $(\bowtie \pi *)$ )

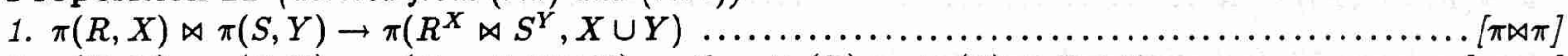

2. $\pi(R, X) \bowtie \pi(S, Y) \rightarrow \pi(R \bowtie S, X \cup Y) \quad$ if $\operatorname{attr}(R) \cap \operatorname{attr}(S) \subset X \cap Y \ldots \ldots \ldots \ldots \ldots \ldots[\pi \bowtie \pi *]$

Proposition 22 (derived from $(\pi \bowtie)$ and $(\pi \bowtie *)$ )

1. $\pi(R \bowtie S, X) \rightarrow \pi(\pi(R, \operatorname{attr}(R) \cap(\operatorname{attr}(S) \cup X)) \bowtie \pi(S, \operatorname{attr}(S) \cap(\operatorname{attr}(R) \cup X)), X) \ldots \ldots \ldots[\pi \bowtie+]$

2. $\pi(R \bowtie S, X) \rightarrow \pi(R, \operatorname{attr}(R) \cap X) \bowtie \pi(S, \operatorname{attr}(S) \cap X) \quad$ if $\quad \operatorname{attr}(R) \cap \operatorname{attr}(S) \subset X \ldots \ldots \ldots[\pi \bowtie \#]$

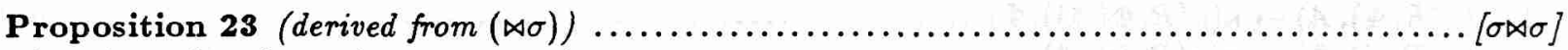

$\sigma(R, A) \bowtie \sigma(S, B) \rightarrow \sigma(R \bowtie S, A \wedge B)$

The next proposition we need for derivation of $(\kappa \bowtie \kappa)$. In general if both $\Phi$ and $\Psi$ are solution sets, the expression $\Phi \cup \Psi$ might not be a solution set. One possible reason is that heads from $\Phi$ also occur as heads from $\Psi$. In this case the $\oplus$ operator can be used to merge the tails in $\Phi$ and the tails in $\Psi$ together:

Proposition 24 (introduction of the merge operator)

$[\kappa \bowtie \kappa *]$

$\kappa(R, \Phi) \bowtie \kappa(S, \Psi) \rightarrow \kappa(\sigma(R \bowtie S, \Phi \oplus \Psi), \Phi) \quad$ if $\quad h \operatorname{var}(\Phi)=h \operatorname{var}(\Psi)$

Proposition 25 (generalization of $(\bowtie \kappa)$ )

$\kappa(R, \Phi) \bowtie \kappa(S, \Psi) \rightarrow$

$\kappa(\sigma(R \bowtie S$,

$\Phi \oplus \Psi \wedge \Phi[\operatorname{attr}(S)] \wedge \Psi\langle h \operatorname{var}(\Phi)\rangle[\operatorname{attr}(R)])$,

$\Phi\langle\operatorname{attr}(S)\rangle \cup \Psi\langle h \operatorname{var}(\Phi)\rangle\langle\operatorname{attr}(R)\rangle)$

Proof: Informally we first explain the construction. In the righthand side of $(\kappa \bowtie \kappa)$ the merge operator $\oplus$ handles the case that there is a solution $x=t \in \Phi$ and a solution $y=s \in \Psi$ with $x=y$, in analogy to rule $(\kappa \bowtie \kappa *)$. Also another case needs to be checked. Suppose there is a solution $x=t \in \Phi$ such that $x \in \operatorname{attr}(S)$. If this solution were put in the computation of the calculate operator, then the resulting expression would be unwellformed. The problem can be handled by recognizing that $x=t$ now 
satisfies the wellformedness conditions of the select operator, viz. $\operatorname{var}(x=t) \subset \operatorname{attr}(R) \cup \operatorname{attr}(S)$. So the restriction operator inserts the solution $x=t$ in the condition of the select operator and the delete operator deletes it from the calculate computation. The symmetric case that there is a solution $x=t \in \Psi$ such that $x \in \operatorname{attr}(R)$, is handled in the same way.

It should be noted that in the construction the expression

$$
\Psi\langle h \operatorname{var}(\Phi)\rangle[\operatorname{attr}(R)]
$$

in the select condition can be replaced by the more simple expression $\Psi[\operatorname{attr}(R)]$ since the following holds:

$\Phi \oplus \Psi \wedge \Phi[\operatorname{attr}(S)] \wedge \Psi\langle h \operatorname{var}(\Phi)\rangle[\operatorname{attr}(R)]=\Phi \oplus \Psi \wedge \Phi[\operatorname{attr}(S)] \wedge \Psi[\operatorname{attr}(R)]$

However this could lead to duplicate use of solutions from $\Psi$ in the select condition and since the rule is to be used for query optimization we want to avoid this duplication.

For a formal derivation of $(\kappa \bowtie \wedge)$ we first observe:

$\Phi \oplus \Psi=\Phi[h \operatorname{var}(\Psi)] \oplus \Psi[h \operatorname{var}(\Phi)]$

Now put:

$$
\begin{array}{ll}
\Phi_{1}=\Phi[\operatorname{attr}(S)] & \boldsymbol{\Psi}_{1}=\Psi[\operatorname{attr}(R)] \\
\boldsymbol{\Phi}_{2}=\Phi[h \operatorname{var}(\Psi)] & \boldsymbol{\Psi}_{2}=\Psi[\operatorname{hvar}(\Phi)] \\
\boldsymbol{\Phi}_{3}=\Phi\langle\operatorname{attr}(S) \cup \operatorname{hvar}(\Psi)\rangle & \boldsymbol{\Psi}_{3}=\Psi\langle\operatorname{attr}(R) \cup h \operatorname{var}(\Phi)\rangle
\end{array}
$$

Both $\Phi$ and $\Psi$ can be obtained as mutually disjoint unions of the above solution sets:

$$
\Phi=\Phi_{1} \cup \Phi_{2} \cup \Phi_{3}, \Psi=\Psi_{1} \cup \Psi_{2} \cup \Psi_{3}
$$

We have:

$\kappa(R, \Phi) \bowtie \kappa(S, \Psi)$

$\rightarrow \kappa\left(R, \Phi_{1} \cup \Phi_{2} \cup \Phi_{3}\right) \bowtie \kappa\left(S, \Psi_{1} \cup \Psi_{2} \cup \Psi_{3}\right)$

$\rightarrow \kappa\left(\kappa\left(\kappa\left(R, \Phi_{1}\right), \Phi_{2}\right), \Phi_{3}\right) \bowtie \kappa\left(\kappa\left(\kappa\left(S, \Psi_{1}\right), \Psi_{2}\right), \Psi_{3}\right)$

$\rightarrow \kappa\left(\kappa\left(\kappa\left(\kappa\left(R, \Phi_{1}\right), \Phi_{2}\right) \bowtie \kappa\left(\kappa\left(S, \Psi_{1}\right), \Psi_{2}\right), \Psi_{3}\right), \Phi_{3}\right)$

$\rightarrow \kappa\left(\kappa\left(\kappa\left(R, \Phi_{1}\right), \Phi_{2}\right) \bowtie \kappa\left(\kappa\left(S, \Psi_{1}\right), \Psi_{2}\right), \Phi_{3} \cup \Psi_{3}\right)$

$\rightarrow \kappa\left(\kappa\left(\sigma\left(\kappa\left(R, \Phi_{1}\right) \bowtie \kappa\left(S, \Psi_{1}\right), \Phi_{2} \oplus \Psi_{2}\right), \Phi_{2}\right), \Phi_{3} \cup \Psi_{3}\right)$

$\rightarrow \kappa\left(\kappa\left(\sigma\left(\kappa\left(R, \Phi_{1}\right) \bowtie \kappa\left(S, \Psi_{1}\right), \Phi \oplus \Psi\right), \Phi_{2}\right), \Phi_{3} \cup \Psi_{3}\right)$

$\rightarrow \kappa\left(\kappa\left(\sigma\left(\sigma\left(R \bowtie \kappa\left(S, \Psi_{1}\right), \Phi_{1}\right), \Phi \oplus \Psi\right), \Phi_{2}\right), \Phi_{3} \cup \Psi_{3}\right)$

$\rightarrow \kappa\left(\kappa\left(\sigma\left(\sigma\left(\sigma\left(R \bowtie S, \Psi_{1}\right), \Phi_{1}\right), \Phi \oplus \Psi\right), \Phi_{2}\right), \Phi_{3} \cup \Psi_{3}\right)$

$\rightarrow \kappa\left(\sigma\left(R \bowtie S, \Phi_{1} \wedge \Psi_{1} \wedge \Phi \oplus \Psi\right), \Phi_{2} \cup \Phi_{3} \cup \Psi_{3}\right)$ $(\sigma \sigma, \sigma \sigma, \kappa \kappa *)$

The last expression yields $(\kappa \bowtie \kappa)$ by backsubstitution of $\Phi_{1}, \Phi_{2}, \Phi_{3}, \Psi_{1}$ and $\Psi_{3}$ and application of (**).

\section{The language PCSJL}

In this section we define a sublanguage PCSJL of UPCSJL together with a normal form for PCSJL. This normal form will be used in the next section for the construction of the UPCSJL normal form.

Definition 26 The language PCSJL consists of expressions constructed from the functions: $\bowtie, \pi, \kappa, \sigma$

Definition 27 A PCSJL normal form is an expression of the form

$$
\pi\left(\kappa\left(\sigma\left(R_{1} \bowtie \ldots \bowtie R_{n}, A\right), \Phi\right), X\right)
$$

where $R_{1}, \ldots, R_{n}$ are base relations.

Another feasible normal form (i.e. format to which all expressions are reducible) exchanges the positions of the select and calculate operators:

$$
\pi\left(\sigma\left(\kappa\left(R_{1} \bowtie \ldots \bowtie R_{n}, \Phi\right), A\right), X\right)
$$

However the disadvantage of this normal form is that unnecessary computations are performed for tuples for which the select condition evaluates to false. Derivation of our normal form below is achieved by applying four unconditional rules of the form $R \rightarrow S$ for each of the primitive four relational operators. 
Proposition 28 The expression $\pi(\kappa(\sigma(R, A), \Phi), X)$ is wellformed iff:

1. $\operatorname{var}(A) \subset \operatorname{attr}(R)$

2. $\operatorname{tvar}(\Phi) \subset \operatorname{attr}(R)$

3. $\operatorname{hvar}(\Phi) \cap \operatorname{attr}(R)=\emptyset$

4. $X \subset \operatorname{attr}(R) \cup h v a r(\Phi)$

Proposition 29 Consequences of wellformedness of $\pi(\kappa(\sigma(R, A), \Phi), X)$ :

1. $\operatorname{tvar}(\Phi) \cap h \operatorname{var}(\Phi)=\emptyset$

2. $\operatorname{var}(A) \cap h \operatorname{var}(\Phi)=\emptyset$

3. $h \operatorname{var}(\Phi)=(\operatorname{var}(A) \cup \operatorname{var}(\Phi))-\operatorname{attr}(R)$

Proposition 30 (projection rule derived from $(\pi \pi)$ ) $[\pi \pi \kappa \sigma]$ $\pi(\pi(\kappa(\sigma(R, A), \Phi), X), Y) \rightarrow \pi(\kappa(\sigma(R, A), \Phi), Y)$

Proposition 31 (selection rule derived from $(\sigma \pi),(\sigma \kappa)$ and $(\sigma \sigma)$ ) $[\sigma \pi \kappa \sigma]$ $\sigma(\pi(\kappa(\sigma(R, A), \Phi), X), B) \rightarrow \pi(\kappa(\sigma(R, A \wedge \Phi(B)), \Phi), X)$

Proposition 32 (calculation rule derived from $(\kappa \pi)$ and $(\kappa \kappa)$ ) $[\kappa \pi \kappa \sigma]$ $\kappa(\pi(\kappa(\sigma(R, A), \Phi), X), \Psi) \rightarrow \pi\left(\kappa\left(\sigma\left(R^{X}, A^{X}\right), \Phi^{X} \cup \Phi^{X}((\Psi))\right), h v a r(\Psi) \cup X\right)$

Proposition 33 (join rule) $[\pi \kappa \sigma \bowtie \pi \kappa \sigma]$ $\pi(\kappa(\sigma(R, A), \Phi), X) \bowtie \pi(\kappa(\sigma(S, B), \Psi), Y) \rightarrow$ $\pi\left(\kappa\left(\sigma\left(R^{X} \bowtie S^{Y}\right.\right.\right.$, $\left.A^{X} \wedge B^{Y} \wedge \Phi^{X} \oplus \Psi^{Y} \wedge \Phi^{X}\left[\operatorname{attr}\left(S^{Y}\right)\right] \wedge \Psi^{Y}\left\langle\operatorname{hvar}\left(\Phi^{X}\right)\right\rangle\left[\operatorname{attr}\left(R^{X}\right)\right]\right)$, $\left.\left(\Phi^{X}\left\langle\operatorname{attr}\left(S^{Y}\right)\right\rangle \cup \Psi^{Y}\left\langle h \operatorname{var}\left(\Phi^{X}\right)\right\rangle\left\langle\operatorname{attr}\left(R^{X}\right)\right\rangle\right)[X \cup Y]\right)$, $X \cup Y)$

Proof: $\pi(\kappa(\sigma(R, A), \Phi), X) \bowtie \pi(\kappa(\sigma(S, B), \Psi), Y)$

$\rightarrow \pi\left(\kappa\left(\sigma\left(R^{X}, A^{X}\right), \Phi^{X}\right) \bowtie \kappa\left(\sigma\left(S^{Y}, B^{Y}\right), \Psi^{Y}\right), X \cup Y\right)$ $(\pi \bowtie \pi)$

$\rightarrow \pi\left(\kappa\left(\sigma\left(\sigma\left(R^{X}, A^{X}\right) \bowtie \sigma\left(S^{Y}, B^{Y}\right)\right.\right.\right.$, $\left.\Phi^{X} \oplus \Psi^{Y} \wedge \Phi^{X}\left[\operatorname{attr}\left(S^{Y}\right)\right] \wedge \Psi^{Y}\left\langle h \operatorname{var}\left(\Phi^{X}\right)\right\rangle\left[\operatorname{attr}\left(R^{X}\right)\right]\right)$,

$\left.\left.\Phi^{X}\left\langle\operatorname{attr}\left(S^{Y}\right)\right\rangle \cup \Psi^{Y}\left\langle h \operatorname{var}\left(\Phi^{X}\right)\right\rangle\left\langle\operatorname{attr}\left(R^{X}\right)\right\rangle\right), X \cup Y\right)$

$\rightarrow \pi\left(\kappa\left(\sigma\left(\sigma\left(R^{X} \bowtie S^{Y}, A^{X} \wedge B^{Y}\right)\right.\right.\right.$, $\left.\Phi^{X} \oplus \Psi^{Y} \wedge \Phi^{X}\left[\operatorname{attr}\left(S^{Y}\right)\right] \wedge \Psi^{Y}\left\langle h \operatorname{var}\left(\Phi^{X}\right)\right\rangle\left[\operatorname{attr}\left(R^{X}\right)\right]\right)$, $\left.\left.\Phi^{X}\left\langle\operatorname{attr}\left(S^{Y}\right)\right\rangle \cup \Psi^{Y}\left\langle h \operatorname{var}\left(\Phi^{X}\right)\right\rangle\left\langle\operatorname{attr}\left(R^{X}\right)\right\rangle\right), X \cup Y\right)$ $(\sigma \bowtie \sigma)$ $\rightarrow \pi\left(\kappa\left(\sigma\left(R^{X} \bowtie S^{Y}\right.\right.\right.$, $\left.A^{X} \wedge B^{Y} \wedge \Phi^{X} \oplus \Psi^{Y} \wedge \Phi^{X}\left[\operatorname{attr}\left(S^{Y}\right)\right] \wedge \Psi^{Y}\left\langle\operatorname{hvar}\left(\Phi^{X}\right)\right\rangle\left[\operatorname{attr}\left(R^{X}\right)\right]\right)$, $\left.\left.\left(\Phi^{X}\left\langle\operatorname{attr}\left(S^{Y}\right)\right\rangle \cup \Psi^{Y}\left\langle h \operatorname{var}\left(\Phi^{X}\right)\right\rangle\left\langle\operatorname{attr}\left(R^{X}\right)\right\rangle\right)[X \cup Y]\right), X \cup Y\right)$ $(\sigma \sigma, \pi \kappa)$

Proposition 34 Every wellformed relational expression in PCSJL can be transformed into an equivalent wellformed PCSJL normal form.

Proof: Basis: $R \rightarrow \pi(\kappa(\sigma(R$, true $), \emptyset), \operatorname{attr}(R))$.

Induction: rules $(\pi \pi \kappa \sigma),(\sigma \pi \kappa \sigma),(\kappa \pi \kappa \sigma)$ and $(\pi \kappa \sigma \bowtie \pi \kappa \sigma)$.

Note that the normal form is not unique. Especially the renaming of clashing variables is a rich source of equivalent expressions. We conclude this section with some examples of normalization.

\section{Example 8}

$\kappa(\sigma(\pi(\kappa(\sigma(r(v, w), v>w),\{x=v+w\}),\{w, x\}), x>0),\{v=x+2\})$

$\rightarrow \pi\left(\kappa\left(\sigma\left(r\left(u_{1}, w\right), u_{1}+w>0 \wedge u_{1}>w\right),\left\{v=u_{1}+w+2\right\}\right),\{v, w, x\}\right)$

\section{Example 9}

$\pi(\kappa(\sigma(r(w, y, v), w>y),\{x=w+y\}),\{w, x\}) \bowtie \pi(\kappa(\sigma(s(x, z, v), x>z),\{y=x+z\}),\{x, y, z\})$

$\rightarrow \pi\left(\kappa\left(\sigma\left(r\left(w, u_{1}, u_{2}\right) \bowtie s\left(x, z, u_{3}\right), w>u_{1} \wedge x>z \wedge x=w+u_{1}\right),\{y=x+z\}\right),\{w, x, y, z\}\right)$

\section{Example 10}

$\pi(\kappa(\sigma(r(v, w), v>w),\{x=v+w\}),\{v, w, x\}) \bowtie \pi(\kappa(\sigma(s(y, z), y>z),\{x=y+z\}),\{x, y, z\})$

$\rightarrow \pi(\kappa(\sigma(r(v, w) \bowtie s(y, z), v>w \wedge y>z \wedge v+w=y+z),\{x=v+w\}),\{v, w, x, y, z\})$ 


\section{The language UPCSJL}

The next step is the addition of the union operator, yielding the language UPCSJL. The normal form procedure presented in this section heavily relies on the normal form construction for PCSJL expressions as discussed before. By the availability of this construction, derivation of a UPCSJL normal form is more or less straightforward.

Definition 35 The language UPCSJL consists of expressions constructed from the functions: $\bowtie, \cup, \pi, \kappa, \sigma$

Definition 36 (UPCSJL normal form) If $R_{1}, \ldots, R_{n}$ are PCSJL normal forms then the following is a UPCSJL normal form:

$$
R_{1} \cup \ldots \cup R_{n}
$$

Proposition 37 Every wellformed relational expression in UPCSJL can be transformed into an equivalent wellformed UPCSJL normal form.

Proof: It suffices to distinguish the following six cases:

1. Basis: $R \rightarrow \pi(\kappa(\sigma(R$, true $), \emptyset)$, attr $(R))$.

Induction: in the remaining cases, using the construction of Proposition 34, it is assumed that $R_{i}$ and $S_{i}$ are reduced to PCSJL normal form. The expressions $T_{i}$ are the resulting PCSJL normal forms:

2. $\pi\left(R_{1} \cup \ldots \cup R_{n}, X\right) \rightarrow \pi\left(R_{1}, X\right) \cup \ldots \cup \pi\left(R_{n}, X\right) \rightarrow T_{1} \cup \ldots \cup T_{n} \ldots \ldots \ldots \ldots \ldots \ldots \ldots(\pi \cup, \pi \pi \kappa \sigma)$

3. $\sigma\left(R_{1} \cup \ldots \cup R_{n}, X\right) \rightarrow \sigma\left(R_{1}, X\right) \cup \ldots \cup \sigma\left(R_{n}, X\right) \rightarrow T_{1} \cup \ldots \cup T_{n} \ldots \ldots \ldots \ldots \ldots \ldots . . .(\sigma \cup, \sigma \pi \kappa \sigma)$

4. $\kappa\left(R_{1} \cup \ldots \cup R_{n}, X\right) \rightarrow \kappa\left(R_{1}, X\right) \cup \ldots \cup \kappa\left(R_{n}, X\right) \rightarrow T_{1} \cup \ldots \cup T_{n} \ldots \ldots \ldots \ldots \ldots \ldots \ldots(\kappa \cup, \kappa \pi \kappa \sigma)$

5. $\left(R_{1} \cup \ldots \cup R_{n}\right) \bowtie\left(S_{1} \cup \ldots \cup S_{m}\right) \rightarrow R_{1} \bowtie S_{1} \cup \ldots \cup R_{n} \bowtie S_{m} \rightarrow T_{1} \cup \ldots \cup T_{n * m} \ldots(\bowtie \cup, \pi \kappa \sigma \bowtie \pi \kappa \sigma)$

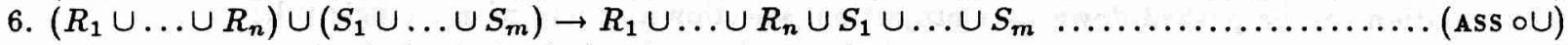

Note that the rules $(\pi \cup),(\sigma \cup),(\kappa \cup),(\bowtie \cup)$ and (ASS $\cup \cup)$ were given in Section 4.2.

\section{Query optimization of UPCSJL normal forms}

Optimization of PSJL expressions (i.e. expressions involving projection, selection and join) is rather well understood (see the literature on query transformations, e.g. [YAN87], [ULL89]). The heuristic of performing selections and projections before joins is effective because the number and size of tuples to be joined can be reduced. If also calculations are applied before joins duplicate computations are avoided. For instance in the next example the variable $v$ is calculated twice instead of four times if the indicated rewriting takes place:

Example 11

$r(x, y):=\{\{x=100, y=3\},\{x=200, y=3\}\}$

$s(y, z):=\{\{y=3, z=10\},\{y=3, z=20\}\}$

$\kappa(r(x, y) \bowtie s(y, z), v=x+1) \rightarrow \kappa(r(x, y), v=x+1) \bowtie s(y, z)$

A procedure for direct optimization of relational expressions, excluding the calculate operator, consists of the following steps (adapted from [ULL89]):

Algorithm 38 (direct optimization)

1. Apply $(\sigma \sigma)$ to separate all compound select conditions:

$$
\sigma\left(R, A_{1} \wedge \ldots \wedge A_{n}\right) \rightarrow \sigma\left(\ldots \sigma\left(R, A_{1}\right) \ldots, A_{n}\right)
$$

2. Apply $(\sigma \sigma *),(\sigma \pi),(\sigma \bowtie)$ and $(\sigma \cup)$ to move selection down.

3. Apply $(\pi \pi),(\pi \bowtie+),(\pi \cup),(\pi \sigma)$ and ( $\pi a t t r)$ to move projection down. Rules $(\pi \pi)$ and ( $(\pi a t t r)$ cause some projections to disappear, while rule $(\pi \sigma)$ splits a projection into two projections, one of which can be migrated downwards if possible.

4. Apply $(\sigma \sigma),(\pi \pi)$ and $(\sigma \pi)$ to combine cascades of selections and projections into a single selection, a single projection or a single projection followed by a single projection. 
A disadvantage of the above algorithm is that the optimization rules are applied on the entire relational expression. An appealing prospect would therefore be first to reduce the number of relational operators, by bringing the expression in UPCSJL normal form. Subsequently it should be possible to apply standard optimization techniques for PSJL expressions on the normal form. Our aim is to give an optimization algorithm for UPCSJL expressions using the heuristic rules of Algorithm 38.

A necessary rewrite rule to accomplish this goal transforms a PCSJL normal form to an expression that contains a 'maximal' PSJL normal form:

Proposition 39 (derived from $(\pi \kappa *))$ $[\pi \kappa \sigma]$ $\pi(\kappa(\sigma(R, A), \Phi), X) \rightarrow \pi(\kappa(\pi(\sigma(R, A), \operatorname{tvar}(\Phi) \cup(\operatorname{attr}(R) \cap X)), \Phi), X)$

In $(\pi \kappa \sigma)$ a projection operator is inserted between calculation and selection such that: 1) the computation $\Phi$ can still be performed, 2) attributes of $R$ that are in $X$ remain available for the outermost projection.

After application of $(\pi \kappa \sigma)$ we need to optimize the newly created PSJL normal form. Algorithm 38 contains the rules $(\pi \sigma)$ and $(\pi \bowtie)$ to move projection down in the relational expression. To avoid that projection is first pushed down over selection and subsequently distributed over the join operator we combine these two steps into a single rule:

Proposition 40 (derived from $(\pi \sigma)$ and $(\pi \bowtie)$ ) $\pi(\sigma(R \bowtie S, A), X) \rightarrow \pi(\sigma(R \bowtie \pi(S, \operatorname{attr}(S) \cap(\operatorname{attr}(R) \cup \operatorname{var}(A) \cup X)), A), X)$ $[\pi \sigma \bowtie]$

In the above rule $(\pi \sigma \bowtie)$ the expression $S$ is projected on the union of: 1) $\operatorname{attr}(R)$ to make sure that the joined attributes remain after projection, 2) $\operatorname{var}(A)$ so that the condition $A$ can be evaluated, 3) $X$ to enforce that necessary attributes of $S$ that are not in $A$ remain after projection.

Once the PSJL expression has been processed also calculation should take part in the optimization. Calculation can be pushed down over projection, selection and join with a single rule:

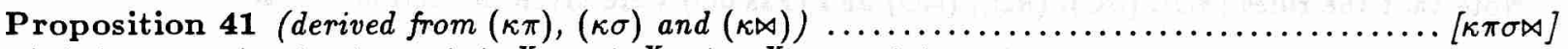
$\kappa(\pi(\sigma(R \bowtie S, A), X), \Phi) \rightarrow \pi\left(\sigma\left(R^{X} \bowtie \kappa\left(S^{X}, \Phi\right), A^{X}\right), h \operatorname{var}(\Phi) \cup X\right) \quad$ if $\quad \operatorname{tvar}(\Phi) \subset \operatorname{attr}(S)$

Efficiency can be gained by generalizing $(\sigma \bowtie),(\pi \sigma \bowtie)$ and $(\kappa \pi \sigma \bowtie)$ to an arbitrary number of joins, so that processing of nested joins is avoided:

Proposition 42 (generalization of $\sigma \bowtie)$ $[\sigma \bowtie \bowtie]$

$\sigma\left(R_{1} \bowtie \ldots \bowtie R_{i} \bowtie \ldots \bowtie R_{n}, A_{1} \wedge \ldots \wedge A_{j} \wedge \ldots \wedge A_{p}\right)$

$\rightarrow \sigma\left(R_{1} \bowtie \ldots \bowtie \sigma\left(R_{i}, A_{j}\right) \bowtie \ldots \bowtie R_{n}, A_{1} \wedge \ldots \wedge A_{j-1} \wedge A_{j+1} \wedge \ldots \wedge A_{p}\right)$

if $\operatorname{var}\left(A_{j}\right) \subset \operatorname{attr}\left(R_{i}\right)$

Proposition 43 (generalization of $\pi \sigma \bowtie$ ) $[\pi \sigma \bowtie \bowtie]$

$\pi\left(\sigma\left(R_{1} \bowtie \ldots \bowtie R_{i} \bowtie \ldots \bowtie R_{n}, A\right), X\right)$

$\rightarrow \pi\left(\sigma\left(R_{1} \bowtie \ldots \bowtie\right.\right.$ $\left.\left.\pi\left(R_{i}, \operatorname{attr}\left(R_{i}\right) \cap\left(\operatorname{attr}\left(R_{1} \bowtie \ldots \bowtie R_{i-1} \bowtie R_{i+1} \bowtie \ldots \bowtie R_{n}\right) \cup \operatorname{var}(A) \cup X\right)\right) \bowtie \ldots \bowtie R_{n}, A\right), X\right)$

Proposition 44 (generalization of $\kappa \pi \sigma \bowtie)$ $[\kappa \pi \sigma \bowtie \bowtie]$ $\kappa\left(\pi\left(\sigma\left(R_{1} \bowtie \ldots \bowtie R_{i} \bowtie \ldots \bowtie R_{n}, A\right), X\right), \Phi_{1} \cup \ldots \cup \Phi_{j} \cup \ldots \cup \Phi_{q}\right)$ $\rightarrow \kappa\left(\pi\left(\sigma\left(R_{1}^{X} \bowtie \ldots \bowtie \kappa\left(R_{i}^{X}, \Phi_{j}\right) \bowtie \ldots \bowtie R_{n}^{X}, A^{X}\right), \operatorname{hvar}\left(\Phi_{j}\right) \cup X\right), \Phi_{1} \cup \ldots \cup \Phi_{j-1} \cup \Phi_{j+1} \cup \ldots \cup \Phi_{q}\right)$ if $\operatorname{tvar}\left(\Phi_{j}\right) \subset \operatorname{attr}\left(R_{i}\right)$

Now we combine the above generalized rules into an optimization algorithm for UPCSJL (i.e. PCSJL expressions extended with union) that uses normalization:

Algorithm 45 (normalization before optimization)

1. Bring the expression into UPCSJL normal form: $S_{1} \cup \ldots \cup S_{m}$

2. Apply the following steps $(a),(b),(c)$ on each $S_{j}$ for $j=1, \ldots m$. $S_{j}$ has the form $\pi\left(\kappa\left(\sigma\left(R_{1} \bowtie \ldots \bowtie R_{n}, A\right), \Phi\right), X\right)$ :

(a) Apply $(\pi \kappa \sigma)$ yielding: $\pi\left(\kappa\left(\pi\left(\sigma\left(R_{1} \bowtie \ldots \bowtie R_{n}, A\right), Y\right), \Phi\right), X\right)$

(b) Optimize the subexpression $\pi\left(\sigma\left(R_{1} \bowtie \ldots \bowtie R_{n}, A\right), Y\right)$ : 
i. Apply $(\sigma \sigma)$ to separate select conditions yielding:

$$
\pi\left(\sigma\left(R_{1} \bowtie \ldots \bowtie R_{n}, A_{1} \wedge \ldots \wedge A_{p}\right), Y\right)
$$

ii. Apply $(\sigma \bowtie \bowtie)$ at most $p$ times to move selection down.

iii. Apply $(\pi \sigma \bowtie \bowtie)$ exactly $n$ times to move projection down.

iv. Apply $(\sigma \sigma)$ to combine cascades of selections and ( $\pi$ attr) to eliminate superfluous projections.

(c) Optimize the subexpression $\kappa\left(\pi\left(\sigma\left(R_{1}^{\prime} \bowtie \ldots \bowtie R_{n}^{\prime}, A^{\prime}\right), Y\right), \Phi\right)$ resulting from the previous step:

i. Apply $(\kappa \kappa *)$ to separate calculate computations yielding:

$$
\kappa\left(\pi\left(\sigma\left(R_{1}^{\prime} \bowtie \ldots \bowtie R_{n}^{\prime}, A^{\prime}\right), Y\right), \Phi_{1} \cup \ldots \cup \Phi_{q}\right)
$$

ii. Apply $(\kappa \pi \sigma \bowtie \bowtie)$ at most $q$ times to move calculation down.

iii. Apply $(\kappa \kappa *)$ to combine cascades of calculations.

\section{Conclusions}

In this paper we have defined UPCSJL, a language with expressions built up using the operations union, projection, selection, join and calculate. We have shown that a Normal Form Theorem for this language exists, using unconditional rules to reduce arbitrary relational expressions into normal form. In addition we showed that after normalization conditional optimization rules can benefit from the fixed structure of the normal form.

There are several directions for further research in this area. First of all the translation of RL expressions into UPCSJL expressions is to be worked out, making use of a constraint solver: this is currently investigated by the authors. Another interesting point is the existence of a normal form that includes the difference operator. However there seems to be no easy normal form for this case since in general projection does not commute with set difference.

\section{References}

[DATE89] Date, C.J. \& White, C.J., A Guide to DB2, (Third Edition), Addison- Wesley Publishing Company (1989).

[DEN90a] van Denneheuvel, S. \& van Emde Boas, P., The rule language $R L / 1$, A. M. Tjoa \& R. Wagner (eds.), Database and Expert Systems Applications, Proc. of the International Conference, Vienna, Austria, Aug 1990, Springer Verlag Wien, 381-387, (1990).

[DEN90b] van Denneheuvel, S., Kwast K. \& Renardel de Lavalette G. R., A normal form for PCSJ expressions, Computing Science in the Netherlands, nov 1990, (to appear).

[HANS88] Hansen, M.R., Algebraic Optimization of Recursive Database Queries, Information Systems and Operations Research 26 286-298, (1988).

[HANS89] Hansen, M.R., Hansen, B.S., Lucas, P. \& van Emde Boas, P., Integrating Relational Databases and Constraint Languages, in Comput. Lang. Vol. 14, No. 2, 63-82, (1989).

[LAR85] Larson, P.A., Yang, H.Z., Computing Queries from Derived Relations, Proc. of the 11th Intl. Conf. on VLDB, 259-269, (1985).

[ULL89] Ullman, J.D., Principles of Data and Knowledge - Base Systems, Volume II: The New Technologies, Computer Science Press, (1989).

[VEMD86a] van Emde Boas, P., RL, a Language for Enhanced Rule Bases Database Processing, Working Document, Rep IBM Research, RJ 4869 (51299), (1986).

[VEMD86b] van Emde Boas, P., A semantical model for the integration and modularization of rules, Proceedings MFCS 12, Bratislava, August 1986, Springer Lecture Notes in Computer Science 233, 78-92, (1986). 
[VEMD86c] van Emde Boas, H. \& van Emde Boas, P., Storing and Evaluating Horn-Clause Rules in a Relational Database, IBM J. Res. Develop. 30 (1), 80-92, (1986).

[YAN87] Yang, H. Z., Larson, P. A., Query Transformations for PSJ-queries, Proc. of the 13 th Int. Conf. on VLDB, Brighton, 245-254, (1987). 


\section{Logic Group Preprint Series \\ Department of Philosophy \\ University of Utrecht \\ Heidelberglaan 2 \\ 3584 CS Utrecht \\ The Netherlands}

1 C.P.J. Koymans, J.L.M. Vrancken, Extending Process Algebra with the empty process, September 1985

2 J.A. Bergstra, A process creation mechanism in Process Algebra, September 1985

3 J.A. Bergstra, Put and get, primitives for synchronous unreliable message passing, October 1985

4 A. Visser, Evaluation, provably deductive equivalence in Heyting's arithmetic of substitution instances of propositional formulas, November 1985

5 G.R. Renardel de Lavalette, Interpolation in a fragment of intuitionistic propositional logic, January 1986

6 C.P.J. Koymans, J.C. Mulder, A modular approach to protocol verification using Process Algebra, April 1986

7 D. van Dalen, F.J. de Vries, Intuitionistic free abelian groups, April 1986

8 F. Voorbraak, A simplification of the completeness proofs for Guaspari and Solovay's $R$, May 1986

9 H.B.M. Jonkers, C.P.J. Koymans \& G.R. Renardel de Lavalette, A semantic framework for the COLD-family of languages, May 1986

10 G.R. Renardel de Lavalette, Strictheidsanalyse, May 1986

11 A. Visser, Kunnen wij elke machine verslaan? Beschouwingen rondom Lucas' argument, July 1986

12 E.C.W. Krabbe, Naess's dichotomy of tenability and relevance, June 1986

$13 \mathrm{H}$. van Ditmarsch, Abstractie in wiskunde, expertsystemen en argumentatie, Augustus 1986

14 A. Visser, Peano's Smart Children, a provability logical study of systems with built-in consistency, October 1986

15 G.R. Renardel de Lavalette, Interpolation in natural fragments of intuitionistic propositional logic, October 1986

16 J.A. Bergstra, Module Algebra for relational specifications, November 1986

17 F.P.J.M. Voorbraak, Tensed Intuitionistic Logic, January 1987

18 J.A. Bergstra, J. Tiuryn, Process Algebra semantics for queues, January 1987

19 F.J. de Vries, A functional program for the fast Fourier transform, March 1987

20 A. Visser, A course in bimodal provability logic, May 1987

21 F.P.J.M. Voorbraak, The logic of actual obligation, an alternative approach to deontic logic, May 1987

22 E.C.W. Krabbe, Creative reasoning in formal discussion, June 1987

23 F.J. de Vries, A functional program for Gaussian elimination, September 1987

24 G.R. Renardel de Lavalette, Interpolation in fragments of intuitionistic propositional logic, October 1987 (revised version of no: 15)

25 F.J. de Vries, Applications of constructive logic to sheaf constructions in toposes, October 1987

26 F.P.J.M. Voorbraak, Redeneren met onzekerheid in expertsystemen, November 1987

27 P.H. Rodenburg, D.J. Hoekzema, Specification of the fast Fourier transform algorithm as a term rewriting system, December 1987 
28 D. van Dalen, The war of the frogs and the mice, or the crisis of the Mathematische Annalen, December 1987

29 A. Visser, Preliminary Notes on Interpretability Logic, January 1988

30 D.J. Hoekzema, P.H. Rodenburg, Gauß elimination as a term rewriting system, January 1988

31 C. Smoryński, Hilbert's Programme, January 1988

32 G.R. Renardel de Lavalette, Modularisation, Parameterisation, Interpolation, January 1988

33 G.R. Renardel de Lavalette, Strictness analysis for POLYREC, a language with polymorphic and recursive types, March 1988

34 A. Visser, A Descending Hierarchy of Reflection Principles, April 1988

35 F.P.J.M. Voorbraak, A computationally efficient approximation of Dempster-Shafer theory, April 1988

36 C. Smoryński, Arithmetic Analogues of McAloon's Unique Rosser Sentences, April 1988

37 P.H. Rodenburg, F.J. van der Linden, Manufacturing a cartesian closed category with exactly two objects, May 1988

38 P.H. Rodenburg, J.L.M.Vrancken, Parallel object-oriented term rewriting : The Booleans, July 1988

39 D. de Jongh, L. Hendriks, G.R. Renardel de Lavalette, Computations in fragments of intuitionistic propositional logic, July 1988

40 A. Visser, Interpretability Logic, September 1988

41 M. Doorman, The existence property in the presence of function symbols, October 1988

42 F. Voorbraak, On the justification of Dempster's rule of combination, December 1988

43 A. Visser, An inside view of EXP, or: The closed fragment of the provability logic of $I \Delta_{0}+\Omega_{1}$, February 1989

44 D.H.J. de Jongh \& A. Visser, Explicit Fixed Points in Interpretability Logic, March 1989

45 S. van Denneheuvel \& G.R. Renardel de Lavalette, Normalisation of database expressions involving calculations, March 1989

46 M.F.J. Drossaers, A Perceptron Network Theorem Prover for the Propositional Calculus, July 1989

47 A. Visser, The Formalization of Interpretability, August 1989

48 J.L.M. Vrancken, Parallel Object Oriented Term Rewriting : a first implementation in Pool2, September 1989

49 G.R. Renardel de Lavalette, Choice in applicative theories, September 1989

50 C.P.J. Koymans \& G.R. Renardel de Lavalette, Inductive definitions in COLD-K, September 1989

51 F. Voorbraak, Conditionals, probability, and belief revision (preliminary version), October 1989

52 A. Visser, On the $\Sigma_{1}^{0}$-Conservativity of $\Sigma_{1}^{0}$-Completeness, October 1989

53 G.R. Renardel de Lavalette, Counterexamples in applicative theories with choice, January 1990

54 D. van Dalen, L.E.J. Brouwer. Wiskundige en Mysticus, June 1990

55 F. Voorbraak, The logic of objective knowledge and rational belief, September 1990

56 J.L.M. Vrancken, Reflections on Parallel and Functional Languages, September 1990

57 A. Visser, An inside view of EXP, or: The closed fragment of the provability logic of $I \Delta_{0}+\Omega_{1}$, revised version with new appendices, October 1990

58 S. van Denneheuvel, K. Kwast, G.R. Renardel de Lavalette, E. Spaan, Query optimization using rewrite rules, October 1990 